\title{
Pemphigus Vulgaris: Present and Future Therapeutic Strategies
}

\author{
Dario Didona ${ }^{1}$, Giovanni Paolino², Giovanni Di Zenzo ${ }^{3}$, Biagio Didona ${ }^{3}$, Riccardo \\ Pampena ${ }^{4}$, Matteo Riccardo Di Nicola ${ }^{2}$, Santo Raffaele Mercuri ${ }^{2}$
}

1 Department of Dermatology and Allergology, Philipps University, Marburg, Germany
2 Unit of Dermatology, IRCCS San Raffaele Hospital, Milan, Italy
3 IDI-IRCCS, Rome, Italy
4 Centro Oncologico ad Alta Tecnologia Diagnostica-Dermatologica, Azienda Unità Sanitaria Locale-IRCCS di Reggio Emilia, Italy

Key words: anti-CD20 antibodies, neonatal Fc receptor (FcRn), pemphigus, rituximab, treatment

Citation: Didona D, Paolino G, Di Zenzo G, Didona B, Pampena R, Di Nicola MR, Mercuri SR. Pemphigus vulgaris: present and future therapeutic strategies. Dermatol Pract Concept. 2022;12(1): e2022037. DOI: https://doi.org/10.5826/dpc.1201a37

Accepted: August 18, 2021; Published: January 2022

Copyright: $@ 2022$ Didona et al. This is an open-access article distributed under the terms of the Creative Commons Attribution-NonCommercial License (BY-NC-4.0), https://creativecommons.org/licenses/by-nc/4.0/, which permits unrestricted noncommercial use, distribution, and reproduction in any medium, provided the original authors and source are credited.

Funding: None.

Competing interests: None.

Authorship: All authors have contributed significantly to this publication. DD and GP have equally contributed to the article and share co-first authorship.

Corresponding author: Matteo Riccardo Di Nicola, Unit of Dermatology, IRCCS San Raffaele Hospital, Milan, Italy.

E-mail: dinicola.matteo@hsr.it

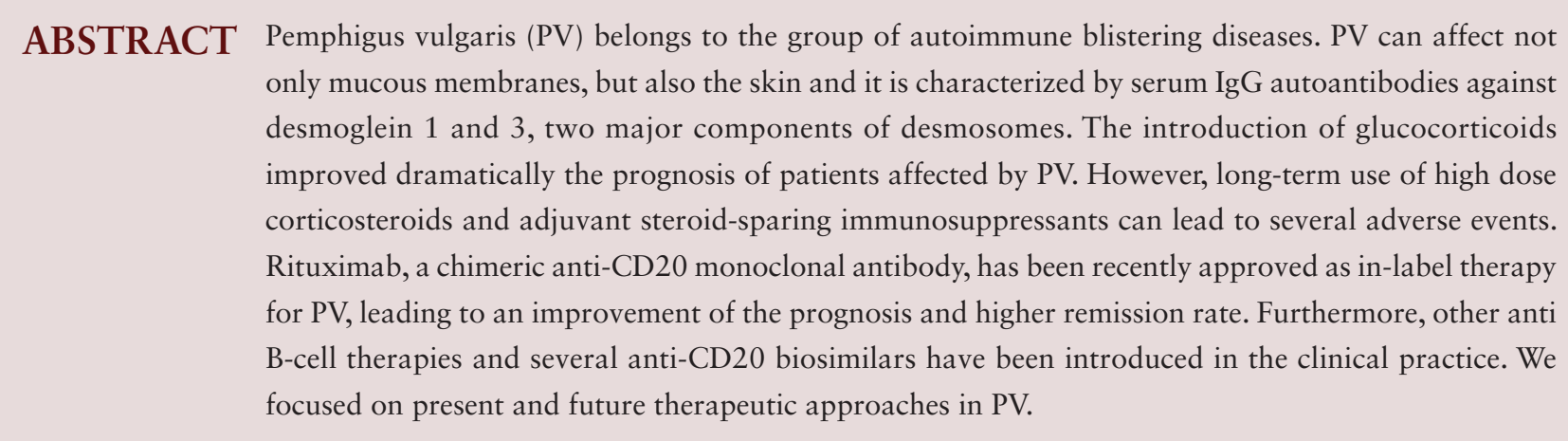

\section{Introduction}

Pemphigus vulgaris (PV) belongs to autoimmune blistering diseases and it is characterized by flaccid blisters and erosions, that can involve not only the skin, but also mucous membranes [1]. Three main forms of pemphigus are described: PV, pemphigus foliaceus (PF), and paraneoplastic pemphigus [1-6]. 


\section{Methods}

We conducted a review to identify studies that documented the current therapeutic strategies for pemphigus vulgaris, as well as the future ones. All type of study, in English language, was considered eligible for this review, including case reports and case series. The main search was conducted in the electronic databases of MEDLINE, EMBASE and Cochrane Central Register of Controlled Trials (CENTRAL) from inception to January 2021 using different combinations of the following terms: "pemphigus", "pemphigus vulgaris", "treatment" and "therapy". Additionally, we concluded the manual search by reviewing all relevant citations within the selected and identified articles.

\section{Epidemiology}

PV is the most frequent type of pemphigus [1]. It usually affects people between 50-60 years of age [7]. A female to male ratio of 5.0 was reported in the USA [7]. In the American general population, an annual incidence of $4.2 / 1,000,000$ inhabitants was reported, but it was much higher in the Jewish-American population [7]. This is due to the most prominent expression of specific HLA class II genes in PV patients with Jewish background, such as HLA-DRB1*0402 and HLA-DQB1*0503 [7].

\section{Clinical Features of PV}

PV usually arises with painful and refractory oral erosions (Figure 1) [1]. Furthermore, other mucous membranes can be affected [1]. Most of patients also develop flaccid skin blisters that rapidly evolve into oozing erosions (Figure 2) [1]. Rarely, pemphigus patients show a clinical and serological transition from PV to PF or conversely. This phenomenon could be due to the epitope spreading, a process of diversification of B- or T-cell responses from the initial dominant epitope to a second one [8].

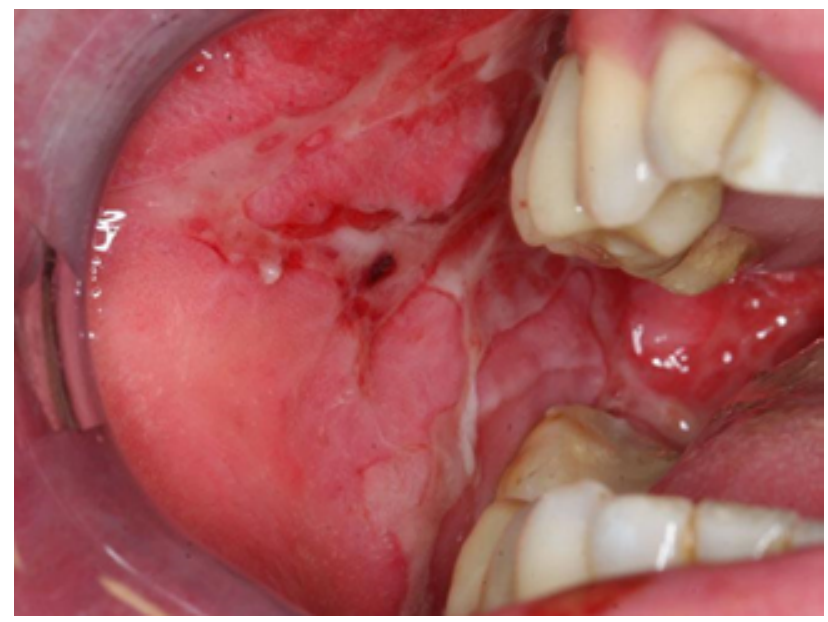

Figure 1. Large erosions of the oral mucosa.

\section{Pathogenesis}

Cutaneous desmoglein-1 (Dsg1) can be expressed in the whole epidermis, cutaneous Dsg3 is typically found in the lower epidermis, while in the mucosa Dsg1 and Dsg3 are located in the whole squamous layer, with a higher expression of Dsg3 [9]. Therefore, PV patients who show only anti-Dsg1 immunoglobulin $\mathrm{G}(\mathrm{IgG})$ serum antibodies develop only skin blisters, and, in the case of detectable anti-Dsg3 IgG serum antibodies, the clinical phenotype is characterized by erosions or ulcerations of mucosal membranes [10]. Furthermore, the production of both anti-Dsg1 and anti-Dsg3 IgG serum autoantibodies provokes skin and mucosal lesions [10].

Evidence suggests that anti-Dsg1 and anti-Dsg3 autoantibodies are responsible for a loss of cell-cell adhesion between keratinocytes $[11,12]$. The most important targets for autoantibodies in PV are represented by the extracellular domains of Dsg $[13,14]$. Further mechanisms can also lead to acantholysis in PV, such as Dsg endocytosis and desmosome disassembly $[15,16]$, and intercellular stretch at non-acantholytic cell layers caused by pathogenic autoantibodies $[17,18]$. In addition, non-Dsg IgG serum autoantibodies have been reported as important in PV pathogenesis, including those directed against desmocollins, mitochondria, pemphaxin, and alpha-9 acetylcholine receptor $[13,19]$.

\section{Diagnosis of PV}

The diagnosis of PV requires not only compatible clinical features, but evidence of pathological features of involved skin and the presence of autoantibodies by direct immunofluorescence microscopy of non-affected skin. Indirect immunofluorescence microscopy, enzyme-linked immunosorbent assay and other techniques have a confirmatory role [5].

The most important pathological feature is the intraepidermal acantholysis [20]. Direct immunofluorescence of non-affected skin detects $\mathrm{IgG}$ and proteins of complement C3 (C3) on epidermal keratinocytes (Figure 3) [20,21]. Indirect

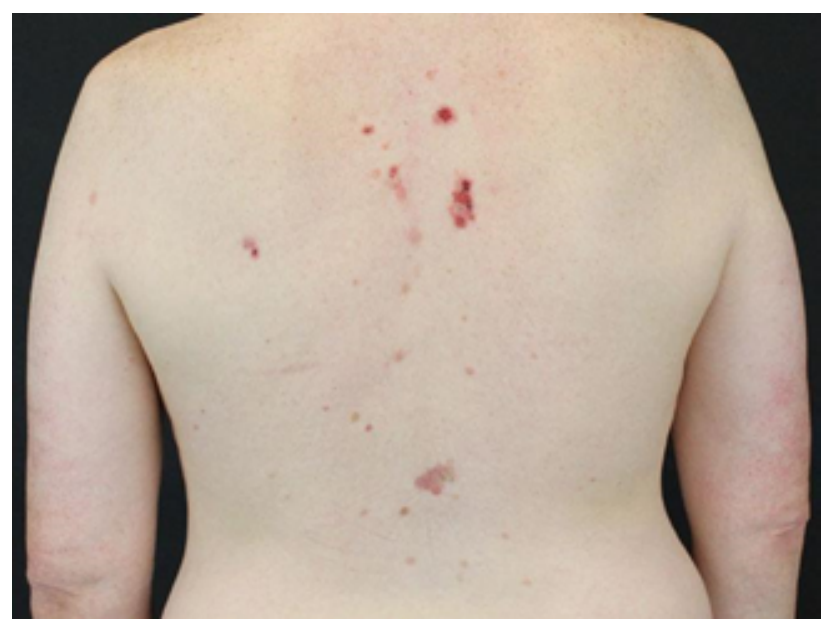

Figure 2. Multiple erosions on the back of this male patient. 


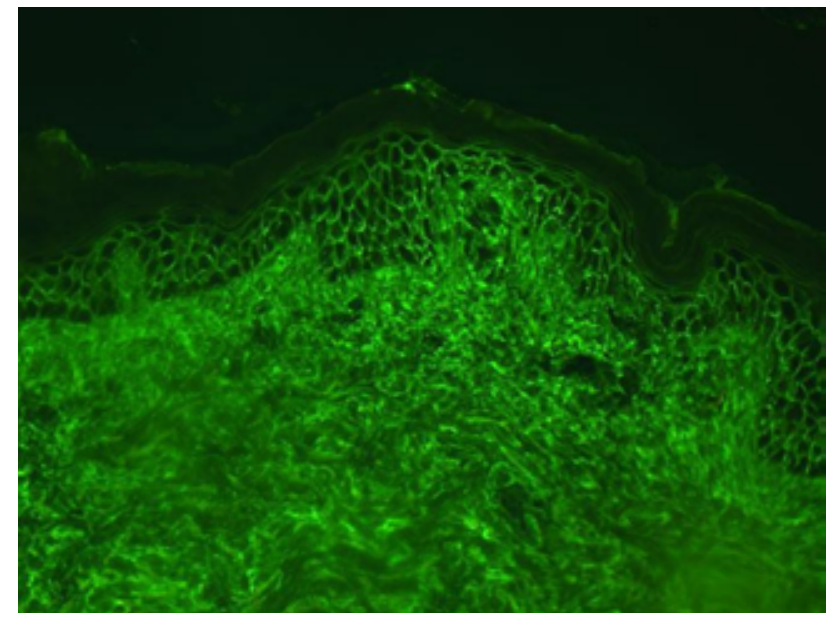

Figure 3. Deposition of IgG and/or C3 on the surface of epidermal keratinocytes detected by direct immunofluorescence. $\mathrm{C} 3=$ proteins of complement C3; IgG = Immunoglobulin G.

immunofluorescence on monkey esophagus detects a fishnet pattern due to IgG antibodies reactivity to cell membrane of epithelial or epidermal cells [20].

\section{Current Therapies \\ Corticosteroids}

Prednisolone is usually administered as initial therapy in PV in association with immunosuppressive agents, such as azathioprine (AZA) and mycophenolate mofetil (MMF), or anti-CD20 monoclonal antibodies [1]. In patients with several comorbidities and in those who cannot undergo a therapy with anti-CD20 monoclonal antibodies or immunosuppressive agents, prednisolone as monotherapy is still recommended as first-line therapy [1]. Nevertheless, plenty of side effects have been described after prolonged corticosteroid (CS) therapy, including severe infections, secondary impairment of adrenal glands, osteoporosis, hyperglycemia, and hypertension [1].

\section{$A Z A$}

AZA downregulates purine metabolism, and blocks the synthesis of DNA, RNA, and proteins. In addition, AZA causes a reduction of Langerhans cells and monocytes, and reduces the activity of T- and B-lymphocytes [1]. Furthermore, AZA blocks T-helper-cell dependent responses of B-cells [1]. AZA dosage should be adapted to thiopurine-methyltransferase activity, the enzyme responsible for AZA metabolism. Adverse events (AEs) are reported in up to $30 \%$ of patients, including nausea, pancreatitis, diarrhea, aphthous stomatitis, and maculopapular rash [1]. Pancytopenia and hepatotoxicity are reported as severe AEs [1].

\section{$M M F$}

MMF leads to a suppression of the immune system by a selective blockade of inosine monophosphate dehydrogenase, that produces a downregulation of the pathway of purine synthesis in T- and B-cells [1]. Because of its mode of action, MMF represents a safer CS-sparing drug compared to other immunosuppressive drugs [1]. Moderate gastrointestinal AEs are frequently reported [1]. In addition, MMF can increase the risk of hematologic malignancies, skin basal cell, and squamous cell carcinoma [1].

\section{Cyclophosphamide}

Cyclophosphamide (CYP) is an alkylating prodrug [1]. It is converted in the liver into 2 active metabolites, which cause cell death through the downregulation of DNA replication. CYP blocks the release of cytokines and reduces the lymphocytic inflammation [1]. It is recommended as a rescue drug, since its administration is characterized by several AEs, such as nausea, fatigue, pancytopenia, and alopecia [1]. A severe complication of CYP treatment is hemorrhagic cystitis, which can be avoided with the administration of adequate fluid intake and sodium 2-mercaptoethane sulfonate [1]. CYP administration can cause transitional cell carcinoma of the bladder [1]. In addition, transient or lasting impairment of gonadal function has been reported [1].

\section{Rituximab}

Rituximab (RTX) is a chimeric monoclonal anti-CD20 antibody, that targets CD20, a transmembrane receptor, expressed at several stages of the B-cell maturation [22]. RTX causes B-cell depletion through different mechanisms: 1) direct induction of apoptosis; 2) complement-dependent cytotoxicity; 3) antibody-dependent cytotoxicity; 4) antibody-dependent phagocytosis; and 5) trogocytosis [23,24]. The last mechanism is characterized by the elimination of RTX-CD20 complexes by macrophages, that causes cell death by a still unknown mechanism [25].

PV patients on RTX can develop opportunistic infections, such as Pneumocystis jirovecii pneumonia [23], but it is still unclear whether PV on RTX may receive a Pneumocystisjirovecii prophylaxis [26]. Furthermore, reactivation of hepatitis B and C and tuberculosis could be possible [23]. Side effects related to RTX administration are represented mostly by type I allergic reaction and cytokine release syndrome [23]. Furthermore, late AEs include serum sickness and toxic epidermal necrolysis [23,27].

The optimal RTX dose in PV is still under debate. Two main protocols have been proposed: 2 intravenous infusions of $1000 \mathrm{mg}$ each 2 weeks apart (rheumatoid arthritis protocol) and 4 infusions of 500-mg each weekly [23,28]. In 2017, a prospective randomized controlled trial that compared RTX combined with CS versus CS alone in patients with newly diagnosed PV showed a significantly higher remission rate off-therapy in the RTX cohort [29]. Furthermore, re-treatment with a single RTX dose of $500 \mathrm{mg}$ after 12 and 
18 months was highly effective in achieving a long-term clinical remission [29].

\section{Ofatumumab}

Ofatumumab is a fully human anti-CD20 monoclonal antibody used as therapy in chronic lymphocytic leukemia. Its target is represented by another CD20 epitope compared to the one targeted by RTX [30]. Ofatumumab has been used for PV patients who developed side effects or loss of response to RTX [31].

\section{Intravenous Immunoglobulin}

Intravenous immunoglobulin (IVIG) is used for immunomodulatory therapy of several inflammatory disorders [32]. The mechanism of action of IVIG is still not completely known, but several modes of action have been proposed $[33,34]$. However, the main mechanism of action is considered the implementation of degradation of immunoglobulins by binding the neonatal of Fc receptor (FcRn) [33,34]. The standard administration schedule is $2 \mathrm{~g} / \mathrm{kg}$ in 5 days ( $400 \mathrm{mg} / \mathrm{kg}$ per day in 5 days) must be kept in mind that IVIG does not show an immunosuppressive activity [32,34]. It can be administered in combination with systemic CS and other immunosuppressants in recalcitrant PV [35].

Side effects were not frequently described [36,37]. Early AEs include headache, nausea, fever, tachycardia, malaise, arthralgia, and dyspnea [36,37]. Late-onset AEs include, aseptic meningitis, acute renal failure, thromboembolic events, and pseudohyponatremia [36,37].

\section{Immunoadsorption}

Through immunoadsorption (IA) IgG were passively removed from systemic circulation [1]. The combination of IA with immunosuppressive therapies is considered an effective treatment for pemphigus patients with severe activity, because IA allows an immediate removal of pathogenic autoantibodies. Infections are still the most frequently complications [1]. IA is considered an effective treatment in patients with severe disease $(>30 \%$ of the body surface or $>25 \%$ of genital or oral mucosa) or with involvement of the conjunctiva or esophagus [1].

\section{Future Therapeutic Approaches CAR-T Cell Therapies}

Chimeric antigen receptor (CAR)-T-cell therapy has been described as promising therapy in hematology [1]. CAR-T cell therapy is a paradigmatic example of adoptive cell transfer therapy. Indeed, autologous T-cells are modified ex-vivo to express a CAR, which leads to a specific targeting of a particular antigen and elimination of the antigen-expressing cells $[38,39]$.

The CARs are composed of 3 domains: 1 ) the extracellular domain, which represents the antigen recognition domain; 2) the transmembrane and hinge domain; 3) the one or more intracellular T-cell signaling domains [39]. In 2016, T-cells were modified to express a chimeric autoantibody receptor (CAAR), which was composed by Dsg 3 fused to a CD137CD3-zeta signaling domains [39]. Desmoglein-3 CAAR-T-cells show a selective cytotoxicity directed to cells with anti-Dsg3 B cell receptors in vitro and destroy Dsg3-specific B-cells in vivo. In a PV mouse model, CAAR-T cells reduced pathogenic IgG antibodies and improved the clinical picture [40].

\section{Anti-Neonatal Fc Receptor (FcRn)}

The FcRn is formed by the MHC class I-like heavy chain and the $\beta_{2}$-microglobulin light chain [41]. It has played a central role in the homeostasis of IgG. Indeed, the IgG-FcRn complex avoids the degradation of $\mathrm{IgG}$, leading to a recycle and release of IgG [42,43]. In a Knockout Mouse for FcRn, loss of cell-cell adhesion by passive transfer of antibodies against Dsg was not evident [44]. Furthermore, it was reported that blocking FcRn impaired the capability of PV to determine acantholysis [45]. A randomized, double-blind, placebo-controlled study with efgartigimod, a human IgG1-derived Fc fragments bound to FcRn, reported the efficacy of the drug in reducing the IgG titer in up to $75 \%$ of patients [46].

\section{Conclusions}

PV remains a therapeutic challenge for clinicians. Several therapeutic options are currently available. However, finding a specific treatment for a particular patient is not easy. Therefore, knowledge and management of multiple therapeutic choices for patients with PV play a pivotal role in better patient management.

\section{References}

1. Didona D, Maglie R, Eming R, Hertl M. Pemphigus: Current and Future Therapeutic Strategies. Front Immunol. 2019;10:1418. DOI: 10.3389/fimmu.2019.01418. PMID: 31293582; PMCID: PMC6603181.

2. Solimani F, Maglie R, Pollmann R, et al. Thymoma-Associated Paraneoplastic Autoimmune Multiorgan Syndrome-From Pemphigus to Lichenoid Dermatitis. Front Immunol. 2019;10:1413. DOI: 10.3389/fimmu.2019.01413. PMID: 31293579; PMCID: PMC6598597.

3. Didona D, Di Zenzo G, Joly P. Paraneoplastic autoimmune multiorgan syndrome. Ital J Dermatol Venerol. 2021;156(2):174-183. DOI: 10.23736/S0392-0488.20.06675-4. PMID: 33070576.

4. Didona D, Fania L, Didona B, Eming R, Hertl M, Di Zenzo G. Paraneoplastic Dermatoses: A Brief General Review and an Extensive Analysis of Paraneoplastic Pemphigus and Paraneoplastic Dermatomyositis. Int J Mol Sci. 2020;21(6):2178. DOI: 10.3390/ ijms21062178. PMID: 32245283; PMCID: PMC7139382.

5. Solimani F, Meier K, Zimmer CL, Hashimoto T. Immune serological diagnosis of pemphigus. G Ital Dermatol Venereol. 2021;156(2):151-160. DOI: 10.23736/S2784-8671.20.06788-7. 
6. Paolino G, Didona D, Magliulo G, et al. Paraneoplastic Pemphigus: Insight into the Autoimmune Pathogenesis, Clinical Features and Therapy. Int J Mol Sci. 2017;18(12):2532. DOI: 10.3390/ ijms18122532. PMID: 29186863; PMCID: PMC5751135.

7. Kridin K, Zelber-Sagi S, Bergman R. Pemphigus Vulgaris and Pemphigus Foliaceus: Differences in Epidemiology and Mortality. Acta Derm Venereol. 2017;97(9):1095-1099. DOI: 10.2340/00015555-2706. PMID: 28536732.

8. Didona D, Di Zenzo G. Humoral Epitope Spreading in Autoimmune Bullous Diseases. Front Immunol. 2018;9:779. DOI: 10.3389/fimmu.2018.00779. PMID: 29719538; PMCID: PMC5913575.

9. Hanakawa Y, Amagai M, Shirakata Y, et al. Differential effects of desmoglein 1 and desmoglein 3 on desmosome formation. $J$ Invest Dermatol. 2002;119(6):1231-1236. DOI: 10.1046/j.15231747.2002.19648.x. PMID: 12485422.

10. Kitajima Y. 150(th) anniversary series: Desmosomes and autoimmune disease, perspective of dynamic desmosome remodeling and its impairments in pemphigus. Cell Commun Adhes. 2014;21(6):269-280. DOI: 10.3109/15419061.2014.943397. PMID: 25078507.

11. Kasperkiewicz M, Ellebrecht CT, Takahashi H, et al. Nat Rev Dis Primers. 2017;3:17026. DOI: 10.1038/nrdp.2017.26. PMID: 28492232 ; PMCID: PMC5901732.

12. Schmidt E, Kasperkiewicz M, Joly P. Pemphigus. Lancet. 2019;394(10201):882-894. DOI: 10.1016/S01406736(19)31778-7. PMID: 31498102.

13. Di Zenzo G, Di Lullo G, Corti D, et al. Pemphigus autoantibodies generated through somatic mutations target the desmoglein-3 cis-interface. J Clin Invest. 2012;122(10):3781-3790. DOI: 10.1172/JCI64413. PMID: 22996451; PMCID: PMC3461925.

14. Tsunoda K, Ota T, Aoki M, et al. Induction of pemphigus phenotype by a mouse monoclonal antibody against the amino-terminal adhesive interface of desmoglein 3. J Immunol. 2003;170(4):21702178. DOI: 10.4049/jimmunol.170.4.2170. PMID: 12574390.

15. Calkins CC, Setzer SV, Jennings JM, et al. Desmoglein endocytosis and desmosome disassembly are coordinated responses to pemphigus autoantibodies. J Biol Chem. 2006;281(11):7623-7634. DOI: 10.1074/jbc.M512447200. PMID: 16377623.

16. Mao X, Choi EJ, Payne AS. Disruption of desmosome assembly by monovalent human pemphigus vulgaris monoclonal antibodies. J Invest Dermatol. 2009;129(4):908-918. DOI: 10.1038/ jid.2008.339. PMID: 19037235; PMCID: PMC2743719.

17. Sokol E, Kramer D, Diercks GFH, et al. Large-Scale Electron Microscopy Maps of Patient Skin and Mucosa Provide Insight into Pathogenesis of Blistering Diseases. J Invest Dermatol. 2015;135(7):1763-1770. DOI: 10.1038/jid.2015.109. PMID: 25789704.

18. Oktarina DA, van der Wier G, Diercks GF, Jonkman MF, Pas HH. IgG-induced clustering of desmogleins 1 and 3 in skin of patients with pemphigus fits with the desmoglein nonassembly depletion hypothesis. Br J Dermatol. 2011;165(3):552-562. DOI: 10.1111/j.1365-2133.2011.10463.x. PMID: 21692763.

19. Di Lullo G, Calabresi V, Mariotti F, Zambruno G, Lanzavecchia A, Di Zenzo G. Identification of a Novel Non-desmoglein Autoantigen in Pemphigus Vulgaris. Front Immunol. 2019;10:1391. DOI: 10.3389/fimmu.2019.01391. PMID: 31275324; PMCID: PMC6593111.

20. Hoffmann K, Hertl M, Sitaru C. Molekulare Diagnostik der blasenbildenden Autoimmundermatosen [Molecular diagnosis of autoimmune dermatoses]. Hautarzt. 2016;67(1):33-39. DOI: 10.1007/s00105-015-3723-9. PMID: 26612472.

21. Witte M,Zillikens D, Schmidt E. Diagnosis of Autoimmune Blistering Diseases. Front Med (Lausanne). 2018;5:296. DOI: 10.3389/ fmed.2018.00296. PMID: 30450358; PMCID: PMC6224342.

22. Musette P, Bouaziz JD. B Cell Modulation Strategies in Autoimmune Diseases: New Concepts. Front Immunol. 2018;9:622. DOI: 10.3389/fimmu.2018.00622. PMID: 29706952; PMCID: PMC5908887.

23. Hebert V, Joly P. Rituximab in pemphigus. Immunotherapy. 2018;10(1):27-37. DOI: 10.2217/imt-2017-0104. PMID: 29064314.

24. Hennerici T, Pollmann R, Schmidt T, et al. Increased Frequency of T Follicular Helper Cells and Elevated Interleukin-27 Plasma Levels in Patients with Pemphigus. PLoS One. 2016;11(2):e0148919. DOI: 10.1371/journal.pone.0148919. PMID: 26872212; PMCID: PMC4752242.

25. Boross P, Jansen JH, Pastula A, van der Poel CE, Leusen JH. Both activating and inhibitory $\mathrm{Fc}$ gamma receptors mediate rituximab-induced trogocytosis of CD20 in mice. Immunol Lett. 2012;143(1):44-52. DOI: 10.1016/j.imlet.2012.01.004. PMID: 22285696.

26. Amber KT, Lamberts A, Solimani F, et al. Determining the Incidence of Pneumocystis Pneumonia in Patients With Autoimmune Blistering Diseases Not Receiving Routine Prophylaxis. JAMA Dermatol. 2017;153(11):1137-1141. DOI: 10.1001/jamadermatol.2017.2808. PMID: 28854309; PMCID: PMC5710438.

27. Didona D, Paolino G, Garcovich S, Caposiena Caro RD, Didona B. Successful use of etanercept in a case of toxic epidermal necrolysis induced by rituximab. J Eur Acad Dermatol Venereol. 2016;30(10):e83-e84. DOI: 10.1111/jdv.13330. PMID: 26428058. Form

28. Murrell DF, Peña S, Joly P, et al. Diagnosis and management of pemphigus: Recommendations of an international panel of experts. J Am Acad Dermatol. 2020;82(3):575-585.e1. DOI: 10.1016/j.jaad.2018.02.021. PMID: 29438767; PMCID: PMC7313440.

29. Joly P, Maho-Vaillant M, Prost-Squarcioni C, et al. First-line rituximab combined with short-term prednisone versus prednisone alone for the treatment of pemphigus (Ritux 3): a prospective, multicentre, parallel-group, open-label randomised trial. Lancet. 2017;389(10083):2031-2040. DOI: 10.1016/S01406736(17)30070-3. PMID: 28342637.

30. Cang S, Mukhi N, Wang K, Liu D. Novel CD20 monoclonal antibodies for lymphoma therapy. J Hematol Oncol. 2012;5:64. DOI: 10.1186/1756-8722-5-64. PMID: 23057966; PMCID: PMC3479003.

31. Rapp M, Pentland A, Richardson C. Successful Treatment of Pemphigus Vulgaris With Ofatumumab. J Drugs Dermatol. 2018;17(12):1338-1339. PMID: 30586270.

32. Forbat E, Ali FR, Al-Niaimi F. Intravenous immunoglobulins in dermatology. Part 2: clinical indications and outcomes. Clin Exp Dermatol. 2018;43(6):659-666. DOI: 10.1111/ced.13552. PMID: 29774587.

33. Dourmishev LA, Guleva DV, Miteva LG. Intravenous Immunoglobulins: Mode of Action and Indications in Autoimmune and Inflammatory Dermatoses. Int J Inflam. 2016;2016:3523057. DOI: $10.1155 / 2016 / 3523057$. PMID: 26885437; PMCID: PMC4739470. 
34. Forbat E, Ali FR, Al-Niaimi F. Intravenous immunoglobulins in dermatology. Part 1: biological mechanisms and methods of administration. Clin Exp Dermatol. 2018;43(5):513-517. DOI: 10.1111/ced.13553. PMID: 29774583.

35. Amagai M, Ikeda S, Shimizu H, et al. A randomized double-blind trial of intravenous immunoglobulin for pemphigus. J Am Acad Dermatol. 2009;60(4):595-603. DOI: 10.1016/j. jaad.2008.09.052. PMID: 19293008 .

36. Tufan F, Kamali S, Erer B, et al. Safety of high-dose intravenous immunoglobulin in systemic autoimmune diseases. Clin Rheumatol. 2007;26(11):1913-1915. DOI: 10.1007/s10067-007-0694-y. PMID: 17636363.

37. Orbach H, Katz U, Sherer Y, et al. Intravenous Immunoglobulin: Adverse Effects and Safe Administration. Clin Rev Allergy Immunol. 2005;29(3):173-184. DOI: 10.1385/CRIAI:29:3:173. PMID: 16391392.

38. Zhang Q, Ping J, Huang Z, et al. CAR-T Cell Therapy in Cancer: Tribulations and Road Ahead. J Immunol Res. 2020;2020:1924379. DOI: 10.1155/2020/1924379. PMID: 32411789 ; PMCID: PMC7201836.

39. Dai H, Wang Y, Lu X, Han W. Chimeric Antigen Receptors Modified T-Cells for Cancer Therapy. J Natl Cancer Inst. 2016;108(7):djv439. DOI: 10.1093/jnci/djv439. PMID: 26819347; PMCID: PMC4948566.

40. Ellebrecht CT, Bhoj VG, Nace A, et al. Reengineering chimeric antigen receptor $\mathrm{T}$ cells for targeted therapy of autoimmune disease. Science. 2016;353(6295):179-184. DOI: 10.1126/science. aaf6756. PMID: 27365313; PMCID: PMC5343513.
41. Junghans RP, Anderson CL. The protection receptor for IgG catabolism is the beta2-microglobulin-containing neonatal intestinal transport receptor. Proc Natl Acad Sci U S A. 1996;93(11):55125516. DOI: 10.1073/pnas.93.11.5512. PMID: 8643606; PMCID: PMC39277.

42. Goebl NA, Babbey CM, Datta-Mannan A, Witcher DR, Wroblewski VJ, Dunn KW. Neonatal Fc receptor mediates internalization of Fc in transfected human endothelial cells. Mol Biol Cell. 2008;19(12):5490-5505. DOI: 10.1091/mbc.e07-02-0101. PMID: 18843053; PMCID: PMC2592658.

43. Ward ES, Ober RJ. Targeting FcRn to Generate Antibody-Based Therapeutics. Trends Pharmacol Sci. 2018;39(10):892-904. DOI: 10.1016/j.tips.2018.07.007. PMID: 30143244; PMCID: PMC6169532.

44. Li N, Zhao M, Hilario-Vargas J, et al. Complete FcRn dependence for intravenous Ig therapy in autoimmune skin blistering diseases. J Clin Invest. 2005;115(12):3440-3450. DOI: 10.1172/JCI24394. PMID: 16284651; PMCID: PMC1280965.

45. Chen Y, Chernyavsky A, Webber RJ, Grando SA, Wang PH. Critical Role of the Neonatal Fc Receptor (FcRn) in the Pathogenic Action of Antimitochondrial Autoantibodies Synergizing with Anti-desmoglein Autoantibodies in Pemphigus Vulgaris. J Biol Chem. 2015;290(39):23826-23837. DOI: 10.1074/jbc.M115.668061. PMID: 26260795; PMCID: PMC4583029.

46. Ulrichts P, Guglietta A, Dreier T, et al. Neonatal Fc receptor antagonist efgartigimod safely and sustainably reduces IgGs in humans. J Clin Invest. 2018;128(10):4372-4386. DOI: 10.1172/JCI97911. PMID: 30040076; PMCID: PMC6159959. 